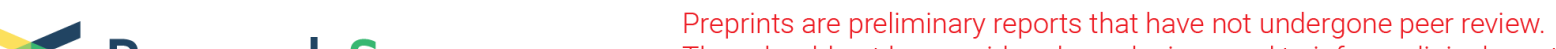

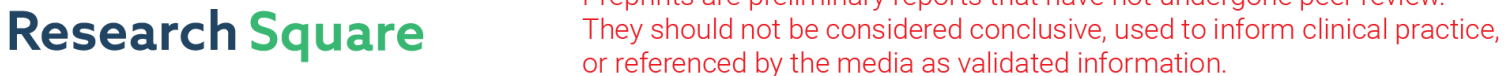

\section{Twist engineering of the two-dimensional magnetism in double bilayer chromium triiodide homostructures}

Hongchao Xie

University of Michigan https://orcid.org/0000-0001-8525-9852

Xiangpeng Luo

University of Michigan

Gaihua Ye

Texas Tech University

Zhipeng Ye

Texas Tech University

\section{Haiwen Ge}

Texas Tech University

\section{Suk Hyun Sung}

University of Michigan-Ann Arbor https://orcid.org/0000-0002-7491-1988

\section{Emily Rennich}

University of Michigan

Shaohua Yan

Renmin University of China

Yang Fu

Renmin University of China

Shang-Jie Tian

Renmin University of China https://orcid.org/0000-0002-3412-4656

Hechang Lei

Renmin University of China https://orcid.org/0000-0003-0850-8514

Robert Hovden

University of Michigan https://orcid.org/0000-0002-3403-8803

\section{Kai Sun}

University of Michigan-Ann Arbor https://orcid.org/0000-0001-9595-7646

Rui He

Texas Tech University https://orcid.org/0000-0002-2368-7269

Liuyan Zhao ( $\nabla$ lyzhao@umich.edu )

University of Michigan-Ann Arbor https://orcid.org/0000-0001-9512-3537 


\section{Letter}

Keywords: twist engineering, two-dimensional (2D) crystalline layers, 2D science, magnetism

Posted Date: March 25th, 2021

DOI: https://doi.org/10.21203/rs.3.rs-356604/v1

License: (c) (i) This work is licensed under a Creative Commons Attribution 4.0 International License. Read Full License

Version of Record: A version of this preprint was published at Nature Physics on December 2nd, 2021. See the published version at https://doi.org/10.1038/s41567-021-01408-8. 


\title{
Twist engineering of the two-dimensional magnetism in double bilayer chromium triiodide homostructures
}

\author{
Hongchao Xie ${ }^{1,+}$, Xiangpeng Luo ${ }^{1,+}$, Gaihua $\mathrm{Ye}^{2,+}$, Zhipeng $\mathrm{Ye}^{2}$, Haiwen $\mathrm{Ge}^{3}$, Suk Hyun Sung ${ }^{4}$, Emily \\ Rennich ${ }^{5}$, Shaohua Yan ${ }^{6}$, Yang Fu ${ }^{6}$, Shangjie Tian ${ }^{6}$, Hechang Lei ${ }^{6}$, Robert Hovden ${ }^{4}$, Kai Sun ${ }^{1}, \mathrm{Rui} \mathrm{He}^{2, *}$, \\ \& Liuyan Zhao ${ }^{1, *}$ \\ ${ }^{1}$ Department of Physics, University of Michigan, 450 Church Street, Ann Arbor, MI, 48109, USA \\ ${ }^{2}$ Department of Electrical and Computer Engineering, Texas Tech University, 910 Boston Avenue, Lubbock, \\ TX, 79409, USA \\ ${ }^{3}$ Department of Mechanical Engineering, Texas Tech University, $27037^{\text {th }}$ Street, Lubbock, TX 79409, USA \\ ${ }^{4}$ Department of Materials Science and Engineering, University of Michigan, 2300 Hayward Street, Ann \\ Arbor, MI, 48109, USA \\ ${ }^{5}$ Department of Mechanical Engineering, University of Michigan, 2350 Hayward Street, Ann Arbor, MI, \\ 48109, USA \\ ${ }^{6}$ Department of Physics and Beijing Key Laboratory of Opto-electronic Functional Materials \& Micro-Nano \\ Devices, Renmin University of China, Beijing, 100872, China \\ *Email: rui.he@ttu.edu; lyzhao@umich.edu \\ ${ }^{+}$These authors contributed equally
}

Twist engineering, or the alignment of two-dimensional (2D) crystalline layers with desired orientations, has led to tremendous success in modulating the charge degree of freedom in heteroand homo-structures, in particular, in achieving novel correlated and topological electronic phases in moiré electronic crystals ${ }^{1,2}$. However, although pioneering theoretical efforts have predicted nontrivial magnetism ${ }^{3,4}$ and magnons ${ }^{5}$ out of twisting 2D magnets, experimental realization of twist engineering spin degree of freedom remains elusive. Here, we leverage the archetypal 2D Ising magnet chromium triiodide $\left(\mathrm{CrI}_{3}\right)$ to fabricate twisted double bilayer homostructures with tunable twist angles and demonstrate the successful twist engineering of 2D magnetism in them. Using linear and circular polarization-resolved Raman spectroscopy, we identify magneto-Raman signatures of a new magnetic ground state that is sharply distinct from those in natural bilayer $(2 \mathrm{~L})$ and four-layer (4L) $\mathrm{CrI}_{3}$. With careful magnetic field and twist angle dependence, we reveal that, for a very small twist angle $\left(\sim 0.5^{\circ}\right)$, this emergent magnetism can be well-approximated by a weighted linear superposition of those of $2 \mathrm{~L}$ and $4 \mathrm{~L} \mathrm{CI_{3 }}$ whereas, for a relatively large twist angle $\left(\sim 5^{\circ}\right)$, it mostly resembles that of isolated $2 \mathrm{~L} \mathrm{CrI}_{3}$. Remarkably, at an intermediate twist angle $\left(\sim 1.1^{\circ}\right)$, its magnetism cannot be simply inferred from the $2 \mathrm{~L}$ and $4 \mathrm{~L}$ cases, because it lacks sharp spin-flip transitions that are present in $2 \mathrm{~L}$ and $4 \mathrm{~L} \mathrm{CrI}_{3}$ and features a dramatic Raman circular dichroism that is absent in natural $2 \mathrm{~L}$ and $4 \mathrm{~L}$ ones. Our results demonstrate the possibility of designing and controlling the spin degree of freedom in 2D magnets using twist engineering. 
Moiré superlattice forms when two vertically stacked atomic crystals are rotated with respect to each other, enabling a powerful venue to design and tailor the physical properties of 2D materials, including electronic, phononic, and magnetic ones. So far, it has achieved fruitful results in controlling the charge degree of freedom (DoF) and realizing novel quantum phenomena in both single-particle electronic states and two-particle excitonic states. Outstanding examples include the creation of flat electronic bands ${ }^{1,2}$ that leads to various strongly correlated ${ }^{6-10}$ and topological ${ }^{11-13}$ phases for the former case, and the introduction of deep confinement potential that localizes excitonic states to realize moiré excitons ${ }^{14,15}$, exciton Mott insulators ${ }^{16}$, and quantum emitters ${ }^{17}$ for the latter. Recently, it has also shown significant impacts on modulating the lattice DoF and induced lattice reconstructions ${ }^{18,19}$, renormalized phonons ${ }^{20}$, and moiré phonons ${ }^{21}$. In sharp contrast, the potential and power of moiré superlattices in controlling the spin DoF and engineering the magnetic properties have remained as a pristine area experimentally, despite a few pioneering theoretical predictions including noncollinear magnetism ${ }^{3}$, topological magnetism ${ }^{4}$, moiré magnon bands ${ }^{5}$, and one-dimensional magnons ${ }^{5}$.

Newly discovered layered magnets have greatly expanded the library of two-dimensional (2D) materials and provided exciting possibilities for the exploration and exploitation of the intrinsic spin DoF at the atomically thin limit ${ }^{22-24}$. Among the 2D magnetic atomic crystals discovered thus far, atomically thin chromium trihalides, $\mathrm{CrX}_{3}(\mathrm{X}=\mathrm{Cl}, \mathrm{Br}$, and I) have attracted extensive interest as an archetype $2 \mathrm{D}$ magnet platform for hosting a plethora of novel 2D magnetic phenomena ${ }^{25-31}$ and exhibiting versatile tunability with external stimuli ${ }^{32-36}$. In particular, it has been theoretically calculated ${ }^{37}$ and experimentally shown ${ }^{35,36}$ in bilayer (2L) $\mathrm{CrI}_{3}$ that tuning between the monoclinic (AB') and rhombohedral (AB) stacking geometries can lead to magnetic transitions from the layered antiferromagnetic (AFM) to ferromagnetic (FM) order. Such a close relationship between structural stacking symmetries and interlayer magnetism in $\mathrm{CrI}_{3}$ naturally stimulates the curiosity of investigating the magnetism in $\mathrm{CrI}_{3}$ moiré magnets where the interlayer exchange coupling is periodically modulated in both sign (i.e., AFM vs FM) and strength (i.e., magnitude).

Here, we fabricate twisted double bilayer (tDB) $\mathrm{CrI}_{3}$ homostructures with individual $2 \mathrm{~L} \mathrm{CrI}_{3}$ to engineer the inter-2L exchange coupling and investigate the resulting moiré magnetism. Our choice of $2 \mathrm{~L} \mathrm{CrI}_{3}$ as the building block is based on its significantly narrower structural and magnetic phonon linewidths than those of monolayer $\mathrm{CrI}_{3}$, suggesting a much better crystalline and magnetic integrity in $2 \mathrm{~L} \mathrm{CrI}_{3}{ }^{38,39}$. Figure 1a top panel shows a false-color optical image of a typical tDB $\mathrm{CrI}_{3}$ sample that was made by tearing a large piece of $2 \mathrm{~L} \mathrm{CrI}_{3}$ in the bottom panel into two and then stacking them together at a controlled twist angle of $\alpha$ (see Methods). Electron diffraction (Fig. 1b) shows two sets of $1^{\text {st }}$ and $2^{\text {nd }}$ order Bragg peaks for the two $2 \mathrm{~L} \mathrm{CrI}_{3}$ in a tDB $\mathrm{CrI}_{3}$ sample with the targeted $\alpha$ of $1.0^{\circ}$ during fabrication, from which the actual $\alpha$ was 
determined to be $0.9^{\circ}$ on average with a standard deviation of $0.1^{\circ}$ through surveying nine different locations on this sample. The match between targeted and measured values confirms our well control of the twist angle in fabrications, and the small standard deviation suggests the homogeneity of our samples. Dark field transmission electron microscopy (TEM) (Fig. 1c) displays the real-space periodic superstructures with noticeable domain formation. The bright triangles are associated with the strongly coupled regions between two $2 \mathrm{~L} \mathrm{CrI}_{3}$ whereas the dark boundaries represent the decoupled boundaries. Similar reconstructuring has been observed in low-twist angle graphene bilayers ${ }^{19}$ and transition metal dichalcogenide bilayers ${ }^{18}$.

Magneto-Raman spectroscopy can capture the interlayer magnetism in few-layer $\mathrm{CrI}_{3}$, by detecting the unique static magnetism-coupled phonons that break the time-reversal symmetry and have antisymmetric Raman tensors ${ }^{38-42}$, in addition to the conventional pure structural phonons. Figure $1 \mathrm{~d}$ presents representative Raman spectra of $\mathrm{tDB} \mathrm{CrI}_{3}$ with the targeted $\alpha=1.1^{\circ}$ in both the crossed and parallel linear polarization channels at $10 \mathrm{~K}$, featuring key Raman modes in three spectral ranges, 75 to $85 \mathrm{~cm}^{-1}, 95$ to 120 $\mathrm{cm}^{-1}$, and 120 to $133 \mathrm{~cm}^{-1}$. These modes are coarsely comparable to those of few-layer $\mathrm{CrI}_{3}$ among which the Raman modes in the 75 to $85 \mathrm{~cm}^{-1}$ and 120 to $140 \mathrm{~cm}^{-1}$ ranges particularly highlight the contribution from the magnetism-coupled-phonon scattering ${ }^{38}$. In this work, we focus on the 120 to $133 \mathrm{~cm}^{-1}$ range because the Raman modes here could be related to the moire magnetism and are of higher intensity than those in 75 to $85 \mathrm{~cm}^{-1}$. Figure 1e zooms into the Raman spectra in the 120 to $133 \mathrm{~cm}^{-1}$ range and includes data in both linearly crossed and parallel channels with incident polarizations at the horizontal and $45^{\circ}$ rotated directions, i.e., $\theta_{\mathrm{inc}}=0^{\circ}$ and $45^{\circ}$. Clearly, the one primary mode in the parallel channels $\left(\mathrm{U}_{1}^{\mathrm{t}}\right.$ at 129.4 $\left.\mathrm{cm}^{-1}\right)$ and the three modes in the crossed channels $\left(\mathrm{U}_{2,3,4}^{\mathrm{t}}\right.$ at $129.0 \mathrm{~cm}^{-1}, 127.4 \mathrm{~cm}^{-1}$, and $\left.126.3 \mathrm{~cm}^{-1}\right)$ all and individually show no polarization dependence, confirming that Raman modes in the crossed (parallel) channels correspond to antisymmetric (fully symmetric) Raman tensors, thus break (preserve) the timereversal symmetry, and correspond to the static magnetism-coupled (pure structural) phonon contribution, similar to those in natural few-layer $\mathrm{CrI}_{3}{ }^{38}$. On the other hand, the triplet lineshape of the $\mathrm{tDB} \mathrm{CrI}_{3} \mathrm{Raman}$ spectra in the crossed channel shows a clear distinction from those of $2 \mathrm{~L}$ and $4 \mathrm{~L} \mathrm{CrI}_{3}$ with a single $\left(\mathrm{U}_{2}^{2 \mathrm{~L}}\right.$ at $\left.127.1 \mathrm{~cm}^{-1}\right)$ and two $\left(\mathrm{U}_{2}^{4 \mathrm{~L}}\right.$ at $128.6 \mathrm{~cm}^{-1}$ and $\mathrm{U}_{4}^{4 \mathrm{~L}}$ at $\left.125.7 \mathrm{~cm}^{-1}\right)$ Raman modes, respectively ${ }^{38}$, shown in Fig. 2a. Such a distinction strongly suggests the substantial difference in the magnetism between $\mathrm{tDB} \mathrm{CrI}_{3}$ and natural $2 \mathrm{~L} / 4 \mathrm{~L} \mathrm{CrI}_{3}$.

Having established the Raman signature for the magnetism of $\mathrm{tDB} \mathrm{CrI}_{3}$ and its distinction from those of natural $2 \mathrm{~L} / 4 \mathrm{~L} \mathrm{CrI}_{3}$, we proceed to examine its twist angle dependence. Figure 2a collects Raman spectra over $120-133 \mathrm{~cm}^{-1}$ taken on $\mathrm{tDB} \mathrm{CrI}_{3}$ of selected $\alpha=0.5^{\circ}, 1.1^{\circ}, 2.0^{\circ}$, and $5.0^{\circ}$, as well as $4 \mathrm{~L}$ and $2 \mathrm{~L} \mathrm{CrI}_{3}$, in both the crossed and parallel linear polarization channels at $10 \mathrm{~K}$. We observe that the central mode $\left(U_{3}^{\mathrm{t}}\right)$ in the triplet in the crossed channel increases in intensity and the two modes on its side $\left(\mathrm{U}_{2,4}^{\mathrm{t}}\right)$ decrease as 
the twist angle $\alpha$ increases, showing the trend that the magneto-Raman spectra of $\mathrm{tDB} \mathrm{CrI}_{3}$ evolves from resembling most the natural $4 \mathrm{~L} \mathrm{CrI}_{3}$ at the lowest $\alpha$ to converging towards the $2 \mathrm{~L} \mathrm{CrI}_{3}$ at the highest $\alpha$. This trend is expected because the inter-2L coupling strength in $\mathrm{tDB} \mathrm{CrI}_{3}$ weakens at larger $\alpha$, leading to the parallel stacked $\left(\alpha=0^{\circ}\right)$ tDB $\mathrm{CrI}_{3}$ relaxing to a $4 \mathrm{~L} \mathrm{CrI}_{3}$ flake and the large twist angle ones are equivalent to two decoupled $2 \mathrm{~L} \mathrm{CrI}_{3}$ films. This intensity evolution is further quantitatively summarized in Fig. 2c where the relative intensity ratio $I_{\mathrm{U}_{3}^{\mathrm{t}}} /\left(I_{\mathrm{U}_{2}^{\mathrm{t}}}+I_{\mathrm{U}_{4}^{\mathrm{t}}}\right)$ is plotted against $\alpha$ for $\mathrm{tDB} \mathrm{CrI}_{3}$ and compared with those of $4 \mathrm{~L}$ and $2 \mathrm{~L} \mathrm{CrI}_{3}$, showing a monotonous enhancement with increasing $\alpha$ and further confirming the corresponding reduction of inter-2L coupling at larger $\alpha$. Equally informative is the frequency shift of the Raman modes which is shown in Fig. 2b. At low twist angles $\left(e . g ., \alpha=0.5^{\circ}, 1.1^{\circ}\right)$, the frequencies of $\mathrm{U}_{2}^{\mathrm{t}}$ and $\mathrm{U}_{4}^{\mathrm{t}}$ in $\mathrm{tDB} \mathrm{CrI}_{3}$ match with those of $\mathrm{U}_{2}^{4 \mathrm{~L}}$ and $\mathrm{U}_{4}^{4 \mathrm{~L}}$ in $4 \mathrm{~L} \mathrm{CrI}_{3}$, respectively, whereas the frequency of $\mathrm{U}_{3}^{\mathrm{t}}$ is close to that of $\mathrm{U}_{2}^{2 \mathrm{~L}}$ in $2 \mathrm{~L} \mathrm{CrI}_{3}$ which appears in the crossed channel same as $\mathrm{U}_{3}^{\mathrm{t}}$ does and that of $\mathrm{U}_{3}^{4 \mathrm{~L}}$ in $4 \mathrm{~L} \mathrm{CrI}_{3}$ which is however absent in the crossed channel in contrast to $\mathrm{U}_{3}^{\mathrm{t}}$. As the twist angle increases, the frequencies of $U_{2}^{t}$ and $U_{4}^{t}$ blueshift towards their high-frequency neighbors $U_{1}^{t}$ and $U_{3}^{t}$. Eventually, at large twist angles (e.g., $\alpha=5^{\circ}$ ), the frequencies of $\mathrm{U}_{2,4}^{\mathrm{t}}$ become nearly indistinguishable from $\mathrm{U}_{1,3}^{\mathrm{t}}$ and approaches those of $\mathrm{U}_{1,2}^{2 \mathrm{~L}}$ in $2 \mathrm{~L} \mathrm{CrI}_{3}$. The correspondence of mode frequencies between the fabricated tDB $\mathrm{CrI}_{3}$ and the natural $4 \mathrm{~L} / 2 \mathrm{~L} \mathrm{CrI}_{3}$ reveals that magnetism-coupled-phonon contributions for $\mathrm{U}_{2}^{\mathrm{t}}$ and $\mathrm{U}_{4}^{\mathrm{t}}$ arise from regions with strong inter-2L coupling, resembling the $4 \mathrm{~L}$-like case, whereas that for $\mathrm{U}_{3}^{\mathrm{t}}$ is dominated by the decoupled regions, mimicking the $2 \mathrm{~L}-$ like case. The twist angle dependencies of mode frequencies and the relative intensity ratio both confirm the reduction of inter-2L coupling and the suppression of strongly coupled $2 \mathrm{~L}-2 \mathrm{~L}$ regions in $\mathrm{tDB} \mathrm{CrI}_{3}$ at larger $\alpha$.

Out-of-plane magnetic field $\left(\mathrm{B}_{\perp}\right)$ is known to introduce sharp spin-flip transitions in few-layer $\mathrm{CrI}_{3}$, which is nicely captured in the $\mathrm{B}_{\perp}$ dependence of Raman spectra of the magnetism-coupled phonon scattering ${ }^{38}$. We now proceed to more in-depth investigations of the engineered magnetism in $\mathrm{tDB} \mathrm{CrI}_{3}$ by performing its magnetic field dependencies for selected twist angles and comparing them across one another and with natural $4 \mathrm{~L} / 2 \mathrm{~L} \mathrm{CrI}_{3}$. Figures $3 \mathrm{a}$-e present the false color maps of the $\mathrm{B}_{\perp}$ dependent magneto-Raman spectra for $4 \mathrm{~L} \mathrm{CrI}_{3}, \mathrm{tDB} \mathrm{CrI}_{3}$ at selected twist angles of $\alpha=0.5^{\circ}, 1.1^{\circ}$, and $5.0^{\circ}$, and $2 \mathrm{~L} \mathrm{CrI}_{3}$, respectively, in the linearly crossed channel at $10 \mathrm{~K}$, and Figures $3 \mathrm{f}-\mathrm{j}$ summarize their corresponding $\mathrm{B}_{\perp}$ dependencies of the fitted mode intensities of the magnetism-coupled phonons. We first briefly summarize the evolutions of $\mathrm{U}_{i}^{4 \mathrm{~L} / 2 \mathrm{~L}}$, with $i=1-4$ for $4 \mathrm{~L}$ and $i=1-2$ for $2 \mathrm{~L} \mathrm{CrI}_{3}$, upon increasing $\mathrm{B}_{\perp}$ as a reference for understanding those of $\mathrm{U}_{1-4}^{\mathrm{t}}$ for $\mathrm{tDB} \mathrm{CrI}_{3}$. For $4 \mathrm{~L} \mathrm{CrI}_{3}, \mathrm{U}_{1}^{4 \mathrm{~L}}$ and $\mathrm{U}_{3}^{4 \mathrm{~L}}$ emerge abruptly at $\mathrm{B}_{\mathrm{c} 1}=0.7 \mathrm{~T}$ and then sharply jump upwards and downwards, respectively, at $\mathrm{B}_{\mathrm{c} 2}=1.6 \mathrm{~T}$, whereas $\mathrm{U}_{2}^{4 \mathrm{~L}}$ and $\mathrm{U}_{4}^{4 \mathrm{~L}}$ both experience two consecutive steep drops at $\mathrm{B}_{\mathrm{c} 1}$ and $\mathrm{B}_{\mathrm{c} 2}$, with plateaus of constant intensities outside of $\mathrm{B}_{\mathrm{c} 1}$ 
and $\mathrm{B}_{\mathrm{c} 2}$, for which $\mathrm{B}_{\mathrm{c} 1}$ and $\mathrm{B}_{\mathrm{c} 2}$ correspond to two first-order spin flip transitions for the layered magnetism transiting from $\uparrow \downarrow \uparrow \downarrow$ (layered AFM) first to $\uparrow \downarrow \uparrow \uparrow$ and then to $\uparrow \uparrow \uparrow \uparrow$ (fully polarized FM), respectively ${ }^{25}$, with $\uparrow(\downarrow)$ for out-of-plane magnetic moment aligning up (down). For $2 \mathrm{~L} \mathrm{CrI}_{3}, \mathrm{U}_{1}^{2 \mathrm{~L}}$ appears and $\mathrm{U}_{2}^{2 \mathrm{~L}}$ disappears concurrently at $B_{c}=0.7 T$ with $B_{c}$ for the spin flip transition from $\uparrow \downarrow$ to $\uparrow \uparrow{ }^{25}$.

We now turn to the magnetic field dependencies of $\mathrm{U}_{1-4}^{\mathrm{t}}$ in $\mathrm{tDB} \mathrm{CrI}_{3}$ at very small $\left(\alpha=0.5^{\circ}\right)$ and relatively large $\left(\alpha=5^{\circ}\right)$ twist angles, both of which can be well described by a simple weighted linear superposition of those of $4 \mathrm{~L}$ and $2 \mathrm{~L} \mathrm{CrI}_{3}$. Similar to the $4 \mathrm{~L}$ case (Figs. $3 \mathrm{a}$ and $3 \mathrm{f}$ ), the $0.5^{\circ} \mathrm{tDB} \mathrm{CrI}_{3}$ features two transitions at $\mathrm{B}_{\mathrm{c} 1}=0.7 \mathrm{~T}$ and $\mathrm{B}_{\mathrm{c} 2}=1.6 \mathrm{~T}$ with jumps/drops in $\mathrm{U}_{1-4}^{\mathrm{t}}$ mode intensities near $\mathrm{B}_{\mathrm{c} 1}$ and $\mathrm{B}_{\mathrm{c} 2}$ of the same trends as those in $4 \mathrm{~L} \mathrm{CrI}_{3}$ and plateaus of constant intensities otherwise (Figs. $3 \mathrm{~b}$ and $3 \mathrm{~g}$ ). Different from the $4 \mathrm{~L}$ case, $\mathrm{U}_{3}^{\mathrm{t}}$ of the $0.5^{\circ} \mathrm{tDB} \mathrm{CrI} \mathrm{I}_{3}$ is present even below $\mathrm{B}_{\mathrm{c} 1}$, and the transitions are slightly broadened. As discussed in Fig. 2, $U_{2}^{\mathrm{t}}$ and $\mathrm{U}_{4}^{\mathrm{t}}$ only originate from regions with strong coupling between the two bilayers in $\mathrm{tDB} \mathrm{CrI}_{3}$, whereas $\mathrm{U}_{1}^{\mathrm{t}}$ and $\mathrm{U}_{3}^{\mathrm{t}}$ match in frequency with modes in both coupled and decoupled regions. Thus, the behaviors of $\mathrm{U}_{1-4}^{\mathrm{t}}$ in the $0.5^{\circ} \mathrm{tDB} \mathrm{CrI}_{3}$ can be well accounted for by a weighted add-up of the $\mathrm{B}_{\perp}$ dependencies of $\mathrm{U}_{1-4}^{4 \mathrm{~L}}$ and $\mathrm{U}_{1-2}^{2 \mathrm{~L}}$ that correspond to the contributions from the strongly coupled and the decoupled $2 \mathrm{~L}-2 \mathrm{~L}$ regions, respectively. On the other hand, the $5.0^{\circ} \mathrm{tDB} \mathrm{CrI}_{3}$ in Figs. $3 \mathrm{~d}$ and $3 \mathrm{i}$ nearly replicates the $2 \mathrm{~L} \mathrm{CrI}_{3}$ results in Figs. $3 \mathrm{e}$ and $3 \mathrm{j}$, except a minor remnant in $\mathrm{U}_{3}^{\mathrm{t}}$ above $\mathrm{B}_{\mathrm{c}}=0.7 \mathrm{~T}$. This observation confirms that the $5.0^{\circ} \mathrm{tDB} \mathrm{CrI}_{3}$ is primarily regarded as two decoupled $2 \mathrm{~L} \mathrm{CrI}_{3}$ with only a tiny fraction of coupled regions. Therefore, the magnetism for both the $0.5^{\circ}$ and $5.0^{\circ} \mathrm{tDB} \mathrm{CrI}_{3}$ can be well described by the combinations of those of $4 \mathrm{~L}$ and $2 \mathrm{~L} \mathrm{CrI}_{3}$, which makes sense because at very low twist angles $\left(e . g ., 0.5^{\circ}\right)$, the structure of $\mathrm{tDB} \mathrm{CrI}_{3}$ relaxes to maximize the natural $4 \mathrm{~L}$-like regions whereas at large

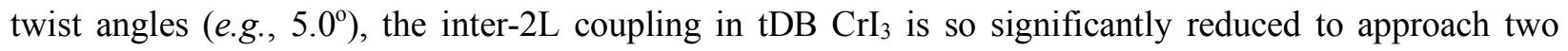
decoupled $2 \mathrm{~L} \mathrm{CrI}_{3}$, similar to many other van der Waals homo- and hetero-structures.

Interestingly, in stark contrast to the $0.5^{\circ}$ and $5.0^{\circ} \mathrm{tDB} \mathrm{CrI}_{3}$, the magnetism in the $1.1^{\circ} \mathrm{tDB} \mathrm{CrI}_{3}$ can no longer be understood simply from the combination of $4 \mathrm{~L}$ and $2 \mathrm{~L} \mathrm{CrI}_{3}$ because its $\mathrm{B}_{\perp}$ dependence features two key behaviors that are not at all present in those of $4 \mathrm{~L}$ and $2 \mathrm{~L} \mathrm{CrI}_{3}$ (Figs. $3 \mathrm{c}$ and $3 \mathrm{~h}$ ). First, around $\mathrm{B}_{\mathrm{c} 1}$ $\approx 0.6 \mathrm{~T}$, the intensities of $\mathrm{U}_{2}^{\mathrm{t}}$ and $\mathrm{U}_{4}^{\mathrm{t}}$ show a dramatic spike feature whereas $\mathrm{U}_{3}^{\mathrm{t}}$ exhibits a sharp dip, instead of the step-like jumps in $4 \mathrm{~L} / 2 \mathrm{~L} \mathrm{CrI}_{3}$. Second, between $\mathrm{B}_{\mathrm{c} 1}$ and $\mathrm{B}_{\mathrm{c} 2} \approx 1.5 \mathrm{~T}$, $\mathrm{U}_{1}^{\mathrm{t}}$ increases gradually until the saturation at $B_{c 2}$ while $U_{3}^{t}$ and $U_{4}^{t}$ decreases slowly towards a finite intensity and zero, respectively, rather than the plateaus of constant intensities in $4 \mathrm{~L} / 2 \mathrm{~L} \mathrm{CrI}_{3}$. These two clear discrepancies between the magnetic field dependencies of the $1.1^{\circ} \mathrm{tDB}$ and $4 \mathrm{~L} / 2 \mathrm{~L} \mathrm{CrI}_{3}$ strongly suggest the emergence of a fundamentally new magnetic phase in the $\mathrm{tDB} \mathrm{CrI}_{3}$ of intermediate twist angles, and therefore the distinction from magnetism in both small and large twist angle $\mathrm{tDB} \mathrm{CrI}_{3}$. 
The anomalous behaviors of the $1.1^{\circ} \mathrm{tDB} \mathrm{CrI}_{3}$ above are further supported by the observation of large Raman circular dichroism at $0 \mathrm{~T}$ which is absolutely zero in $4 \mathrm{~L}$ and $2 \mathrm{~L} \mathrm{CrI}_{3}{ }^{38}$. Figures $4 \mathrm{a}$ and $4 \mathrm{~b}$ show the raw spectra of the $1.1^{\circ}$ tDB $\mathrm{CrI}_{3}$ taken at $0 \mathrm{~T}$ in both the $\mathrm{LL}$ and $\mathrm{RR}$ channels and their corresponding Lorentzian fitting profiles for individual modes, where LL (RR) refers to the incident and scattered light with left- (right-) handed circular polarizations. Here, we note that we could only resolve three $\left(\mathrm{U}_{1,3,4}^{\mathrm{t}}\right)$ out of the four Raman modes because $U_{2}^{t}$ is overwhelmed by the spectrally closest and much stronger $U_{1}^{t}$ in the same co-circularly polarized channels. Clearly, $U_{1}^{t}$ and $U_{3}^{t}$ show substantial differences between the LL and RR channels with opposite relative intensities, whereas $U_{4}^{t}$ is almost helicity independent. Figure $4 \mathrm{c}$ presents the $B_{\perp}$ dependencies of the fitted intensities of $U_{1,3,4}^{t}$ in both co-circularly polarized channels, where the field is swept from $+2 \mathrm{~T}$ to $-2 \mathrm{~T}$ and then back to $+2 \mathrm{~T}$. The two key features highlighted in Fig. 3h, the sharp dip/peak around $B_{c 1}$ for $U_{3}^{t} / U_{4}^{t}$ and the gradual evolution between $B_{c 1}$ and $B_{c 2}$ for all modes, are nicely reproduced in Fig. 4c. In addition, a third feature noticed is that $U_{1}^{t}$ and $U_{3}^{t}$ exhibit notable hysteresis loops below $\mathrm{B}_{\mathrm{c} 1}$, in consistency with their substantial Raman circular dichroism even at $0 \mathrm{~T}$.

Let us point out two important facts before establishing the understanding on these three key features in the $1.1^{\circ} \mathrm{tDB} \mathrm{CrI}_{3}$. First, recent DFT calculations show that both monoclinic and rhombohedral stackings between layers are structurally favorable with nearly identical elastic energies, yet they correspond to distinct interlayer AFM and FM exchange coupling, respectively ${ }^{37}$. In a moiré supercell of the $1.1^{\circ}$ tDB $\mathrm{CrI}_{3}$, both monoclinic and rhombohedral stacking regions take up appreciable amount of areas and feature the strong but opposite magnetic coupling across the 2L-2L interface, leading to the competition and frustration of spins at the boundaries between the monoclinic and rhombohedral stacking regions. Second, our polar magneto-Raman geometry is only sensitive to the out-of-plane components of spins. Although our probe and analysis do not directly account for the in-plane components of spins which are likely to develop from the aforementioned frustrations ${ }^{3,4}$, our results on the out-of-plane magnetic orders in tDB $\mathrm{CrI}_{3}$, at the same time, are compatible with the presence of in-plane spin components.

Therefore, we propose the following model for the magnetism in the $1.1^{\circ} \mathrm{tDB} \mathrm{CrI}_{3}$, where the strongly coupled 2L-2L regions have two types, the monoclinic AFM and the rhombohedral FM inter-2L coupling, and the optimized out-of-plane magnetic order features $\uparrow \downarrow \uparrow \downarrow$ for the monoclinic regions and $\uparrow \uparrow \uparrow \downarrow$ for the rhombohedral regions. As shown in Supplemental Information S1, based on parameters obtained from DFT calculations, this spin configuration has the lowest magnetic energy for intermediate twist angles. Because the $\uparrow \uparrow \uparrow \downarrow$ state generates a net magnetization and contributes an effective out-of-plane magnetic field, which causes the re-orientation of the spins in the outermost layer to require a lower external $B_{\perp}$ in the rhombohedral regions than that in the monoclinic regions, i.e., $B_{c 1}^{R}<B_{c 1}^{M}$ with $R(M)$ denoting the rhombohedral(monoclinic) stacking. Therefore, as a function of $\mathrm{B}_{\perp}$, the magnetic state of the $1.1^{\circ} \mathrm{tDB} \mathrm{CrI}_{3}$ 
undergoes three critical transitions at $\mathrm{B}_{\mathrm{c} 1}^{\mathrm{R}}=0.5 \mathrm{~T}, \mathrm{~B}_{\mathrm{c} 1}^{\mathrm{M}}=0.7 \mathrm{~T}$, and $\mathrm{B}_{\mathrm{c} 2}=1.5 \mathrm{~T}$, corresponding to the spin reorientation in the outermost layer in the rhombohedral and monoclinic regions, and the interior layer in the monoclinic regions, as depicted in Fig. 4d. We now can understand the three key features in the $1.1^{\circ}$ tDB $\mathrm{CrI}_{3}$. First, the dip/peak around $0.6 \mathrm{~T}$ for $\mathrm{U}_{3}^{\mathrm{t}} / \mathrm{U}_{4}^{\mathrm{t}}$ in fact corresponds to the narrow field range between $\mathrm{B}_{\mathrm{c} 1}^{\mathrm{R}}$ and $\mathrm{B}_{\mathrm{c} 1}^{\mathrm{M}}$, where the spin state of $\uparrow \uparrow \uparrow \uparrow$ in the rhombohedral regions provides the least magnetismcoupled phonon contribution for $U_{3,4}^{t}$ modes (see Supplemental Information S2). Second, the gradual $B_{\perp}$ dependencies of all modes between $\mathrm{B}_{\mathrm{c} 1}^{\mathrm{M}}$ and $\mathrm{B}_{\mathrm{c} 2}$ are then due to spin flop towards the $\mathrm{B}_{\perp}$ direction, either by the expansion of the polarized area or by the increase in the out-of-plane components. Third, the Raman circular dichroism of $\mathrm{U}_{1,3}^{\mathrm{t}}$ at $0 \mathrm{~T}$ is from the interference between the individual pure structural and magnetism-coupled phonon contributions both of which are present for the $\uparrow \uparrow \uparrow \downarrow$ state in the rhombohedral regions at $0 \mathrm{~T}$. Thus, the hysteresis of $\mathrm{U}_{1,3}^{\mathrm{t}}$ below $\mathrm{B}_{\mathrm{c} 1}^{\mathrm{R}}$ is naturally explained.

In summary, we have successfully engineered the $2 \mathrm{D}$ magnetism in $\mathrm{tDB} \mathrm{CrI}_{3}$ that exhibits a clear twist angle dependence. Although different from that of $4 \mathrm{~L} / 2 \mathrm{~L} \mathrm{CrI}_{3}$, the magnetism in the very small and large twist angle $\mathrm{tDB} \mathrm{CrI}_{3}$ are dominated by the superpositions between $4 \mathrm{~L}$ - and 2L-like regions, whereas that in the intermediate twist angle features fundamentally new magnetic orders that are absent in $4 \mathrm{~L} / 2 \mathrm{~L} \mathrm{CrI}_{3}$. Our results open up experimental pathways for engineering and understanding 2D moiré magnetism, among which we highlight two immediate opportunities. One is the exploitation of in-plane spin component sensitive experimental techniques, for example, nonlinear optical spectroscopy ${ }^{43-45}$, to develop a comprehensive understanding of moiré magnets where noncollinear spin textures with in-plane components often happen. The other is the exploration of moiré magnets for which the interlayer exchange coupling dominates over the intralayer exchange isotropy in the composing 2D magnets, such as 2D XY-type magnets ${ }^{46,47}$, so that the periodic moiré interlayer exchange interaction is the leading order magnetic energy scale. 


\section{Methods}

Growth of $\mathrm{CrI}_{3}$ single crystals

Single crystals of $\mathrm{CrI}_{3}$ were grown by the chemical vapor transport method. Chromium power (99.99\% purity) and iodine flakes ( $99.999 \%$ purity) in a 1:3 molar ratio were put into a silicon tube with a length of $200 \mathrm{~mm}$ and an inner diameter of $14 \mathrm{~mm}$. The tube was pumped down to $0.01 \mathrm{~Pa}$ and sealed under vacuum, and then placed in a two-zone horizontal tube furnace whose two zones were raised up slowly to $903 \mathrm{~K}$ and $823 \mathrm{~K}$ for 2 days and then held for another 7 days. Shiny, black, plate-like crystals with lateral dimensions of up to several millimeters were obtained from this growth procedure.

Fabrications of 2D $\mathrm{CrI}_{3}$ and $\mathbf{t D B} \mathrm{CrI}_{3}$ Atomically thin $2 \mathrm{D} \mathrm{CrI}_{3}$ flakes were exfoliated in a nitrogen-filled glovebox, and their thickness was first determined by the optical color contrast to select natural $4 \mathrm{~L}$ and $2 \mathrm{~L} \mathrm{CrI}_{3}$ and further confirmed using Raman spectroscopy at $10 \mathrm{~K}$. Using a polymer-stamping technique inside the glovebox, large-size (lateral dimensions greater than $10 \mu \mathrm{m}$ ) $2 \mathrm{~L} \mathrm{CrI}_{3}$ flakes were torn into two parts with similar sizes, one of which was rotated by a well-controlled rotation micrometer for targeted twist angles and then brought down to stack with the remaining half. Both $4 \mathrm{~L} / 2 \mathrm{~L}$ and $\mathrm{tDB} \mathrm{CrI}_{3}$ samples were sandwiched between two few-layer hBN flakes to avoid surface reactions with oxygen and moisture in the ambient environment after taking out from the glovebox. The samples for magneto-Raman spectroscopy measurements were placed onto the $\mathrm{SiO}_{2} / \mathrm{Si}$ substrate, and those for TEM measurements were transferred onto to TEM grids.

Micro-Raman spectroscopy Micro-Raman spectroscopy measurements were carried out using a $632.81 \mathrm{~nm}$ excitation laser with a full width half maximum (FWHM) of $0.85 \mathrm{~cm}^{-1}$, on the resonance with the charge transfer and $\mathrm{Cr}^{3++}{ }^{4} \mathrm{~A}_{2}$ to ${ }^{4} \mathrm{~A}_{1}$ transitions of $\mathrm{CrI}_{3}$ in order to increase the Raman sensitivity. The laser beam on the sample site was focused down to $\sim 3 \mu \mathrm{m}$ FWHM in diameter using a $40 \times$ transmissive objective, and the laser power was kept at about $80 \mu \mathrm{W}$, in order to minimize the local heating effect during measurements. Backscattering geometry was used, where the scattered light was dispersed by a Horiba LabRAM HR Evolution Raman microscope (1800 grooves/mm grating) from Horiba Scientific and detected by a thermoelectric cooled CCD camera from Horiba Scientific. A commercial variable temperature ( $<10 \mathrm{~K}-325 \mathrm{~K}$ ), closed cycle, microscopy cryostat from Cryo Industries of America, Inc was interfaced with the Raman microscope. A commercial cryogen free room-temperature-bore (2"' in diameter and 6.88' long) superconducting magnet from Cryo Industries of America, Inc was used to achieve the variable out-of-plane magnetic field from $0 \mathrm{~T}$ to $2 \mathrm{~T}$. The cryostat cold finger, on which the samples were mounted, was inserted into the center of the room-temperature-bore of the magnet. The linearly polarized magnto-Raman measurements were calibrated by suppressing the Rayleigh line in the linearly crossed channel, so as to overcome the Faraday artifact that is caused by the stray magnetic fields passing through 
the objective. The circularly polarized magneto-Raman measurements were not affected by this Faraday effect, and therefore no corrections were applied.

Transmission Electron Microscopy Crystallographic orientations of the two composing 2L $\mathrm{CrI}_{3}$ flakes in a $\mathrm{tDB} \mathrm{CrI}_{3}$ homostructure were identified by selected area electron diffraction measurements on Thermo Fisher Talos operated at $200 \mathrm{keV}$, equipped with Gatan OneView camera. Each Bragg peak was fitted with a 2D Gaussian to quantify the twist angle in reciprocal space. A total of nine locations were surveyed for the homostructure to develop a statistical confidence in assigning the twist angle and its standard deviation. Figure 1c was generated by averaging DF-TEM images from three $5^{\text {th }}$ order Bragg peaks, spaced $120^{\circ}$ apart to remove anisotropy.

\section{Data availability}

The datasets generated and/or analyzed during the current study are available from the corresponding authors on reasonable request.

\section{Acknowledgement}

L. Zhao acknowledges the support by NSF CAREER Grant No. DMR-174774 and AFOSR YIP Grant No. FA9550-21-1-0065. R. He acknowledges the support by NSF CAREER Grant No. DMR-1760668. K. Sun acknowledges the support by NSF Grant No. NSF-EFMA-1741618. R. Hovden acknowledges the support from W. M. Keck Foundation. This work made use of facilities at Michigan Center for Materials Characterization. H. Lei acknowledges support by the National Key R\&D Program of China (Grant No. 2018YFE0202600, 2016YFA0300504), the National Natural Science Foundation of China (No. 11774423, and 11822412), the Beijing Natural Science Foundation (Grant No. Z200005), and the Fundamental Research Funds for the Central Universities and Research Funds of Renmin University of China (RUC) (Grant No. 18XNLG14 and 19XNLG17, 20XNH062).

\section{Author contributions}

L. Zhao, H. Xie, and X. Luo conceived the ideal and initiated this project; H. Xie fabricated the 4L and 2L

$\mathrm{CrI}_{3}$ and tDB $\mathrm{CrI}_{3}$ homostructures; H. Xie, X. Luo, G. Ye, Z. Ye, and H. Ge carried out the Raman experiments under the supervision of L. Zhao and R. He; S. H. Sung, E. Rennich, and R. Hovden performed the TEM characterizations; S. Yan, Y. Fu, S. Tian, and H. Lei grew the vdW $\mathrm{CrI}_{3}$ bulk single crystals; K. 
Sun performed the theoretical calculations; H. Xie, X. Luo, R. He, and L. Zhao analyzed the data and wrote the manuscript; all authors participated in the discussion of the results.

\section{Competing interests}

The authors declare no competing interests.

\section{Figure captions}

Figure 1. Sample fabrication, TEM and magneto-Raman characterizations of tDB $\mathrm{CrI}_{3}$. a. Falsecolored optical images for a $\mathrm{tDB} \mathrm{CrI}_{3}$ homostructure (top panel) with two composing $2 \mathrm{~L} \mathrm{CrI}_{3}$ outlined with red lines and its original large-size $2 \mathrm{~L} \mathrm{CrI}_{3}$ (bottom panel) with the tearing boundary marked with a white dashed line. b. Electron diffraction patterns of $1^{\text {st }}$ and $2^{\text {nd }}$ order Bragg peaks for a tDB $\mathrm{CrI}_{3}$ with a targeted twist angle $\alpha$ of $1.0^{\circ}$, showing the measured value of $\alpha=0.9^{\circ} \pm 0.1^{\circ}$ based on the survey over nine areas in this tDB $\mathrm{CrI}_{3}$. c. Averaged DF-TEM real space image from three $5^{\text {th }}$ order Bragg peaks showing the hexagonal superlattice network in the $0.9^{\circ} \mathrm{tDB} \mathrm{CrI}_{3}$ in b. d. Full-range Raman spectra taken on a $1.1^{\circ} \mathrm{tDB}$ $\mathrm{CrI}_{3}$ sample in both the crossed (blue) and parallel (gray) linear polarization channels at $10 \mathrm{~K}$. The parallel channel spectrum is scaled by a factor of 0.33 for a better visual comparison with the crossed channel one. e. Zoom-in of Raman spectra over the $120-133 \mathrm{~cm}^{-1}$ frequency range taken on the same $1.1^{\circ} \mathrm{tDB} \mathrm{CrI}_{3}$ sample as in $\mathbf{d}$ in both the parallel (blue) and crossed (gray) channels at two incident polarizations of $\theta_{\text {inc }}$ $=0^{\circ}$ (filled circles and solid lines) and $45^{\circ}$ (open circles and dashed lines) at $10 \mathrm{~K}$. The parallel channel spectra are scaled by a factor of 0.33 .

Figure 2. Twist angle dependence of the magneto-Raman spectra of $\mathbf{t D B} \mathrm{CrI}_{3}$. a. Raman spectra taken on $4 \mathrm{~L} \mathrm{CrI}_{3}, \mathrm{tDB} \mathrm{CrI}_{3}$ homostructures with targeted twist angles of $\alpha=0.5^{\circ}, 1.1^{\circ}, 2.0^{\circ}$, and $5.0^{\circ}$, and $2 \mathrm{~L} \mathrm{CrI}_{3}$ in both the crossed (blue dots and lines) and parallel (gray) channels at $10 \mathrm{~K}$. The Raman spectra are scaled by factors labeled in individual panels. The Raman modes are also marked at their corresponding frequencies, with $\mathrm{U}_{1,2,3,4}^{4 \mathrm{~L}}, \mathrm{U}_{1,2,3,4}^{\mathrm{t}}$, and $\mathrm{U}_{1,2}^{2 \mathrm{~L}}$ for $4 \mathrm{~L}, \mathrm{tDB}$, and $2 \mathrm{~L} \mathrm{CrI}_{3}$. The colored Lorentzian profiles highlight individual modes appearing in the crossed channel, with red (blue) for the central (two side) mode(s) in the triplet spectra of $\mathrm{tDB} \mathrm{CrI}_{3}$ and the mode(s) of $2 \mathrm{~L}(4 \mathrm{~L}) \mathrm{CrI}_{3}$. b. Plot of the fitted Raman frequencies of individual modes in every spectrum shown in a as a function of the twist angle. c. Plot of the 
intensity ratio $I_{\mathrm{U}_{3}^{\mathrm{t}}} /\left(I_{\mathrm{U}_{2}^{\mathrm{t}}}+I_{\mathrm{U}_{4}^{\mathrm{t}}}\right)$ as a function of the twist angle. Error bars correspond to one standard error in fitting the Raman spectra.

Figure 3. Magnetic field dependence of the magneto-Raman spectra of $\mathrm{tDB} \mathrm{CrI}_{3}$ at selected twist angles. a-e. False-colored maps of the $\mathrm{B}_{\perp}$ dependent Raman spectra taken on $4 \mathrm{~L} \mathrm{CrI}_{3}, \mathrm{tDB}^{\mathrm{CrI}} \mathrm{I}_{3}$ homostructures with targeted twist angles $\alpha=0.5^{\circ}, 1.1^{\circ}$, and $5.0^{\circ}$, and $2 \mathrm{~L} \mathrm{CrI}_{3}$, respectively, in the crossed linear polarization channel at $10 \mathrm{~K}$. The blue arrows mark the frequencies of $\mathrm{U}_{1,2,3,4}^{4 \mathrm{~L}}$ for $4 \mathrm{~L} \mathrm{CrI}_{3}$, and the red ones are for those of $U_{1,2}^{2 L}$ for $2 \mathrm{~L} \mathrm{CrI}_{3}$. $\mathbf{f}-\mathbf{j}$. Plots of the fitted mode intensities as a function of $\mathrm{B}_{\perp}$ for the samples in a-e. Open squares are for the fitted values. Thin black lines and solid shaded areas are for the fits to the established model of the magnetism-coupled phonon scattering for the $4 \mathrm{~L}$ (f) and $2 \mathrm{~L}$ (j) $\mathrm{CrI}_{3}$, and to the proposed weighted linear superpositions of $4 \mathrm{~L}$ and $2 \mathrm{~L}$ contributions for the very small $\left(0.5^{\circ}, \mathbf{g}\right)$ and large $\left(5.0^{\circ}, \mathbf{j}\right)$ twist angle $\mathrm{tDB} \mathrm{CrI}_{3}$. Thick gray lines and the striped areas in $\mathbf{h}$ are guide to the eye. Error bars correspond to one standard error in fitting the Raman spectra.

Figure 4. Magnetic circular dichroism for the $1.1^{\circ}$ twist angle tDB $\mathrm{CrI}_{3}$. a. Raman spectra taken in the $\mathrm{LL}$ (red) and RR (blue) co-circularly polarized channels at $10 \mathrm{~K}$ on the $1.1^{\circ} \mathrm{tDB} \mathrm{CrI}_{3}$. b. Fitted Lorentzian profiles for individual modes $\left(U_{1,3,4}^{t}\right)$ in the Raman spectra in a. c. Plots of fitted mode intensities for $U_{1,3,4}^{t}$ as a function of $B_{\perp}$ that is swept from $+2 T$ to $-2 T$ (decreasing $B$, open circle/square for the LL/RR channel) and then to $+2 \mathrm{~T}$ (increasing B, solid circle/square for the LL/RR channel). Three critical magnetic fields, $\mathrm{B}_{\mathrm{c} 1}^{\mathrm{R}}, \mathrm{B}_{\mathrm{c} 1}^{\mathrm{M}}$, and $\mathrm{B}_{\mathrm{c} 2}$, are marked on both the upwards and downwards magnetic fields, and four magnetic field ranges, below $B_{c 1}^{R}, B_{c 1}^{R}$ to $B_{c 1}^{M}, B_{c 1}^{M}$ to $B_{c 2}$, and above $B_{c 2}$ are shaded, respectively, with light green, orange, blue, and red. d. Sketches of the out-of-plane layered magnetism in the two types of strongly coupled 2L-2L regions of a moiré supercell, monoclinic (M) and rhombohedral (R) ones, evolving as the $\mathrm{B}_{\perp}$ increases across $\mathrm{B}_{\mathrm{c} 1}^{\mathrm{R}}, \mathrm{B}_{\mathrm{c} 1}^{\mathrm{M}}$, and $\mathrm{B}_{\mathrm{c} 2}$.

\section{References}

1 Balents, L., Dean, C. R., Efetov, D. K. \& Young, A. F. Superconductivity and strong correlations in moiré flat bands. Nature Physics 16, 725-733, doi:10.1038/s41567-020-0906-9 (2020).

2 Kennes, D. M. et al. Moiré heterostructures as a condensed-matter quantum simulator. Nature Physics 17, 155-163, doi:10.1038/s41567-020-01154-3 (2021).

3 Hejazi, K., Luo, Z.-X. \& Balents, L. Noncollinear phases in moiré magnets. Proceedings of the National Academy of Sciences 117, 10721, doi:10.1073/pnas.2000347117 (2020). 

Letters 18, 7194-7199, doi:10.1021/acs.nanolett.8b03315 (2018).

$5 \quad$ Wang, C., Gao, Y., Lv, H., Xu, X. \& Xiao, D. Stacking Domain Wall Magnons in Twisted van der Waals Magnets. Physical Review Letters 125, 247201, doi:10.1103/PhysRevLett.125.247201 (2020).

6 Cao, Y. et al. Correlated insulator behaviour at half-filling in magic-angle graphene superlattices. Nature 556, 80-84, doi:10.1038/nature26154 (2018).

7 Cao, Y. et al. Unconventional superconductivity in magic-angle graphene superlattices. Nature 556, 43-50, doi:10.1038/nature26160 (2018).

8 Regan, E. C. et al. Mott and generalized Wigner crystal states in $\mathrm{WSe}_{2} / \mathrm{WS}_{2}$ moiré superlattices. Nature 579, 359-363, doi:10.1038/s41586-020-2092-4 (2020).

9 Tang, Y. et al. Simulation of Hubbard model physics in $\mathrm{WSe}_{2} / \mathrm{WS}_{2}$ moiré superlattices. Nature 579, 353-358, doi:10.1038/s41586-020-2085-3 (2020).

10 Kim, K. et al. Tunable moiré bands and strong correlations in small-twist-angle bilayer graphene. Proceedings of the National Academy of Sciences 114, 3364, doi:10.1073/pnas.1620140114 (2017).

11 Nuckolls, K. P. et al. Strongly correlated Chern insulators in magic-angle twisted bilayer graphene. Nature 588, 610-615, doi:10.1038/s41586-020-3028-8 (2020).

12 Wu, S., Zhang, Z., Watanabe, K., Taniguchi, T. \& Andrei, E. Y. Chern insulators, van Hove singularities and topological flat bands in magic-angle twisted bilayer graphene. Nature Materials, doi:10.1038/s41563-020-00911-2 (2021).

13 Chen, G. et al. Tunable correlated Chern insulator and ferromagnetism in a moiré superlattice. Nature 579, 56-61, doi:10.1038/s41586-020-2049-7 (2020).

14 Tran, K. et al. Evidence for moiré excitons in van der Waals heterostructures. Nature 567, 71-75, doi:10.1038/s41586-019-0975-z (2019).

15 Jin, C. et al. Observation of moiré excitons in $\mathrm{WSe}_{2} / \mathrm{WS}_{2}$ heterostructure superlattices. Nature 567, 76-80, doi:10.1038/s41586-019-0976-y (2019).

16 Wang, J. et al. Optical generation of high carrier densities in 2D semiconductor heterobilayers. Science Advances 5, eaax0145, doi:10.1126/sciadv.aax0145 (2019).

17 Seyler, K. L. et al. Signatures of moiré-trapped valley excitons in $\mathrm{MoSe}_{2} / \mathrm{WSe}_{2}$ heterobilayers. Nature 567, 66-70, doi:10.1038/s41586-019-0957-1 (2019).

18 Yoo, H. et al. Atomic and electronic reconstruction at the van der Waals interface in twisted bilayer graphene. Nature Materials 18, 448-453, doi:10.1038/s41563-019-0346-z (2019).

19 Alden, J. S. et al. Strain solitons and topological defects in bilayer graphene. Proceedings of the National Academy of Sciences, 201309394, doi:10.1073/pnas.1309394110 (2013).

20 Quan, J. et al. Phonon renormalization in reconstructed $\mathrm{MoS}_{2}$ moiré superlattices. Nature Materials, doi:10.1038/s41563-021-00960-1 (2021).

21 Lin, M.-L. et al. Moiré Phonons in Twisted Bilayer $\mathrm{MoS}_{2}$. ACS Nano 12, 8770-8780, doi:10.1021/acsnano.8b05006 (2018).

22 Burch, K. S., Mandrus, D. \& Park, J.-G. Magnetism in two-dimensional van der Waals materials. Nature 563, 47-52, doi:10.1038/s41586-018-0631-z (2018).

23 Gong, C. \& Zhang, X. Two-dimensional magnetic crystals and emergent heterostructure devices. Science 363, eaav4450, doi:10.1126/science.aav4450 (2019).

24 Mak, K. F., Shan, J. \& Ralph, D. C. Probing and controlling magnetic states in 2D layered magnetic materials. Nature Reviews Physics 1, 646-661, doi:10.1038/s42254-019-0110-y (2019).

25 Huang, B. et al. Layer-dependent ferromagnetism in a van der Waals crystal down to the monolayer limit. Nature 546, 270-273, doi:10.1038/nature22391 (2017).

26 Wang, Z. et al. Determining the phase diagram of atomically thin layered antiferromagnet $\mathrm{CrCl}_{3}$. Nature Nanotechnology 14, 1116-1122, doi:10.1038/s41565-019-0565-0 (2019).

27 Chen, W. et al. Direct observation of van der Waals stacking-dependent interlayer magnetism. Science 366, 983, doi:10.1126/science.aav1937 (2019). 
Kim, H. H. et al. Evolution of interlayer and intralayer magnetism in three atomically thin chromium trihalides. Proceedings of the National Academy of Sciences 116, 11131, doi:10.1073/pnas.1902100116 (2019).

29 Klein, D. R. et al. Probing magnetism in 2D van der Waals crystalline insulators via electron tunneling. Science 360, 1218, doi:10.1126/science.aar3617 (2018).

30 Jin, W. et al. Observation of the polaronic character of excitons in a two-dimensional semiconducting magnet $\mathrm{CrI}_{3}$. Nature Communications 11, 4780, doi:10.1038/s41467-020-18627$\mathrm{x}(2020)$.

31 Li, S. et al. Magnetic-Field-Induced Quantum Phase Transitions in a van der Waals Magnet. Physical Review X 10, 011075, doi:10.1103/PhysRevX.10.011075 (2020).

32 Jiang, S., Li, L., Wang, Z., Mak, K. F. \& Shan, J. Controlling magnetism in 2D CrI 3 by electrostatic doping. Nature Nanotechnology 13, 549-553, doi:10.1038/s41565-018-0135-x (2018).

33 Huang, B. et al. Electrical control of $2 \mathrm{D}$ magnetism in bilayer $\mathrm{CrI}_{3}$. Nature Nanotechnology 13, 544-548, doi:10.1038/s41565-018-0121-3 (2018).

34 Jiang, S., Shan, J. \& Mak, K. F. Electric-field switching of two-dimensional van der Waals magnets. Nature Materials 17, 406-410, doi:10.1038/s41563-018-0040-6 (2018).

$35 \mathrm{Li}, \mathrm{T}$. et al. Pressure-controlled interlayer magnetism in atomically thin $\mathrm{CrI}_{3}$. Nature Materials $\mathbf{1 8}$, 1303-1308, doi:10.1038/s41563-019-0506-1 (2019).

36 Song, T. et al. Switching 2D magnetic states via pressure tuning of layer stacking. Nature Materials 18, 1298-1302, doi:10.1038/s41563-019-0505-2 (2019).

37 Sivadas, N., Okamoto, S., Xu, X., Fennie, C. J. \& Xiao, D. Stacking-Dependent Magnetism in Bilayer $\mathrm{CrI}_{3}$. Nano Letters 18, 7658-7664, doi:10.1021/acs.nanolett.8b03321 (2018).

38 Jin, W. et al. Tunable layered-magnetism-assisted magneto-Raman effect in a two-dimensional magnet $\mathrm{CrI}_{3}$. Proceedings of the National Academy of Sciences 117, 24664, doi:10.1073/pnas.2012980117 (2020).

39 Jin, W. et al. Raman fingerprint of two terahertz spin wave branches in a two-dimensional honeycomb Ising ferromagnet. Nature Communications 9, 5122, doi:10.1038/s41467-018-075476 (2018).

40 Huang, B. et al. Tuning inelastic light scattering via symmetry control in the two-dimensional magnet $\mathrm{CrI}_{3}$. Nature Nanotechnology 15, 212-216, doi:10.1038/s41565-019-0598-4 (2020).

41 Zhang, Y. et al. Magnetic Order-Induced Polarization Anomaly of Raman Scattering in 2D Magnet $\mathrm{CrI}_{3}$. Nano Letters 20, 729-734, doi:10.1021/acs.nanolett.9b04634 (2020).

42 McCreary, A. et al. Distinct magneto-Raman signatures of spin-flip phase transitions in $\mathrm{CrI}_{3}$. Nature Communications 11, 3879, doi:10.1038/s41467-020-17320-3 (2020).

43 Zhao, L. et al. Evidence of an odd-parity hidden order in a spin-orbit coupled correlated iridate. Nature Physics 12, 32-36, doi:10.1038/nphys3517 (2016).

44 Zhao, L. et al. A global inversion-symmetry-broken phase inside the pseudogap region of $\mathrm{YBa}_{2} \mathrm{Cu}_{3} \mathrm{O}_{\mathrm{y}}$. Nature Physics 13, 250-254, doi:10.1038/nphys3962 (2017).

45 Jin, W. et al. Observation of a ferro-rotational order coupled with second-order nonlinear optical fields. Nature Physics 16, 42-46, doi:10.1038/s41567-019-0695-1 (2020).

46 Kim, K. et al. Suppression of magnetic ordering in XXZ-type antiferromagnetic monolayer NiPS. Nature Communications 10, 345, doi:10.1038/s41467-018-08284-6 (2019).

47 Bedoya-Pinto, A. et al. Intrinsic 2D-XY ferromagnetism in a van der Waals monolayer. arXiv preprint arXiv:2006.07605 (2020). 
Figures
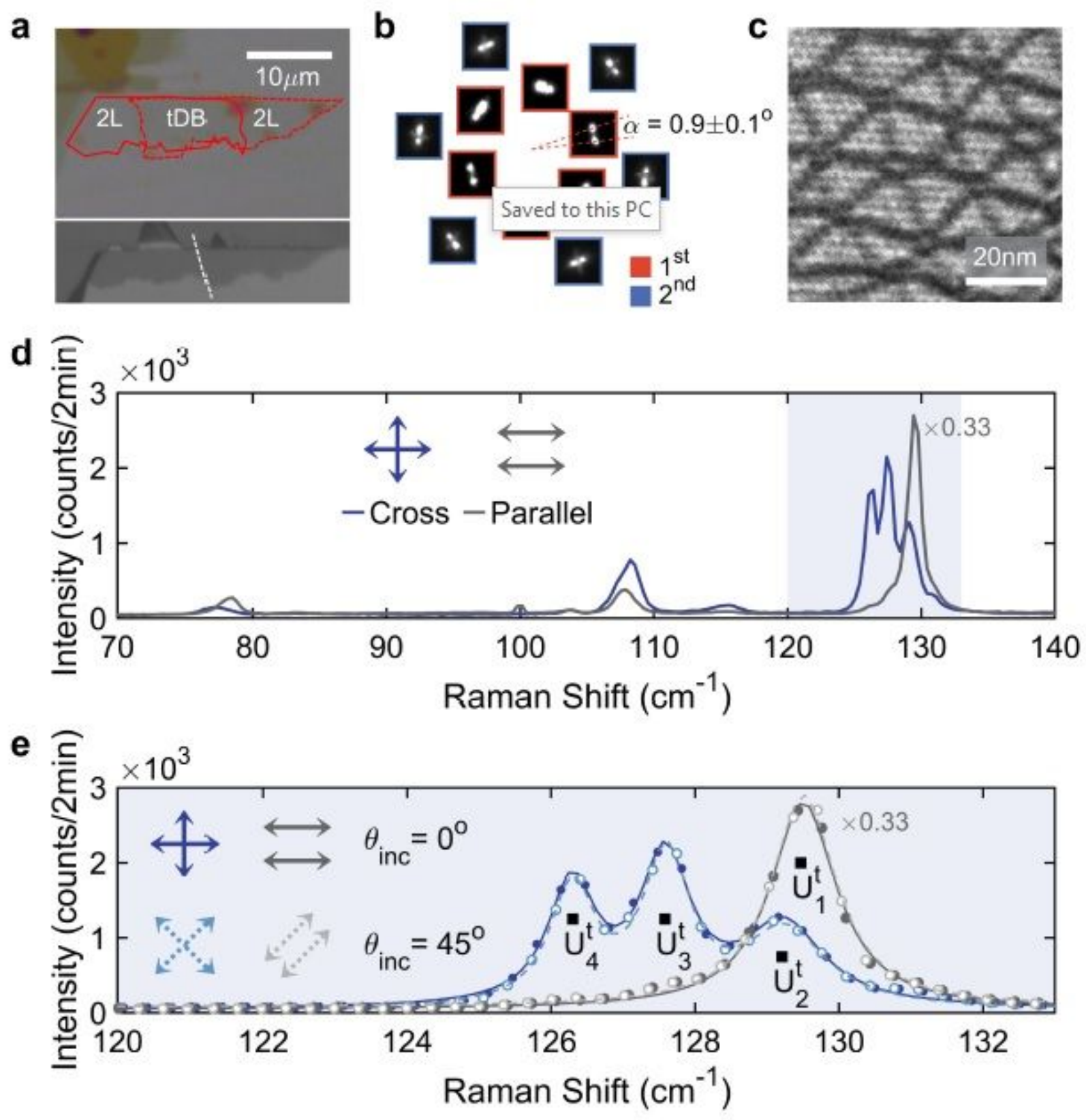

Figure 1

Sample fabrication, TEM and magneto-Raman characterizations of tDB CrI3. (see Manuscript file for full figure legend) 

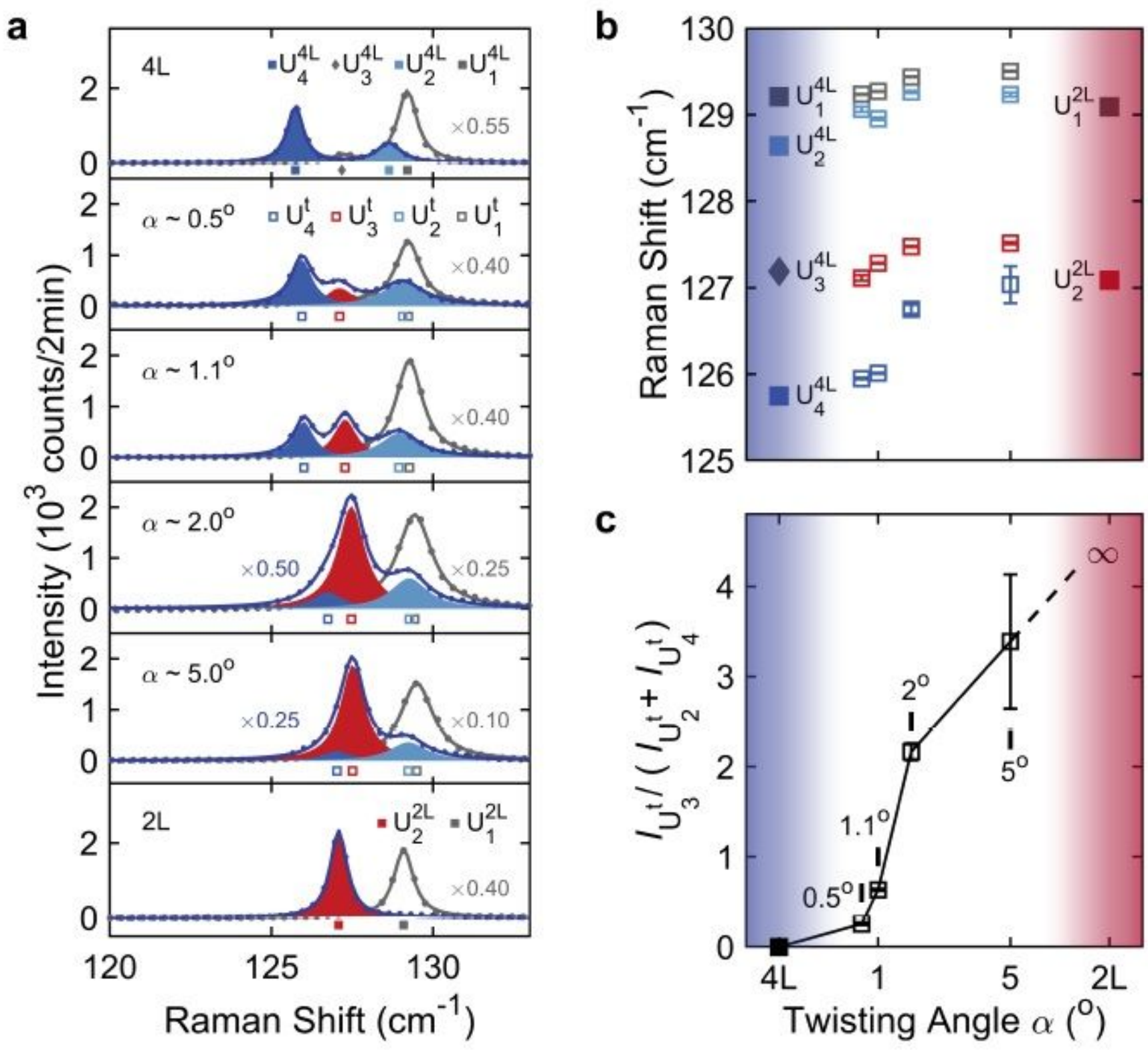

Figure 2

Twist angle dependence of the magneto-Raman spectra of tDB Crl3. (see Manuscript file for full figure legend) 

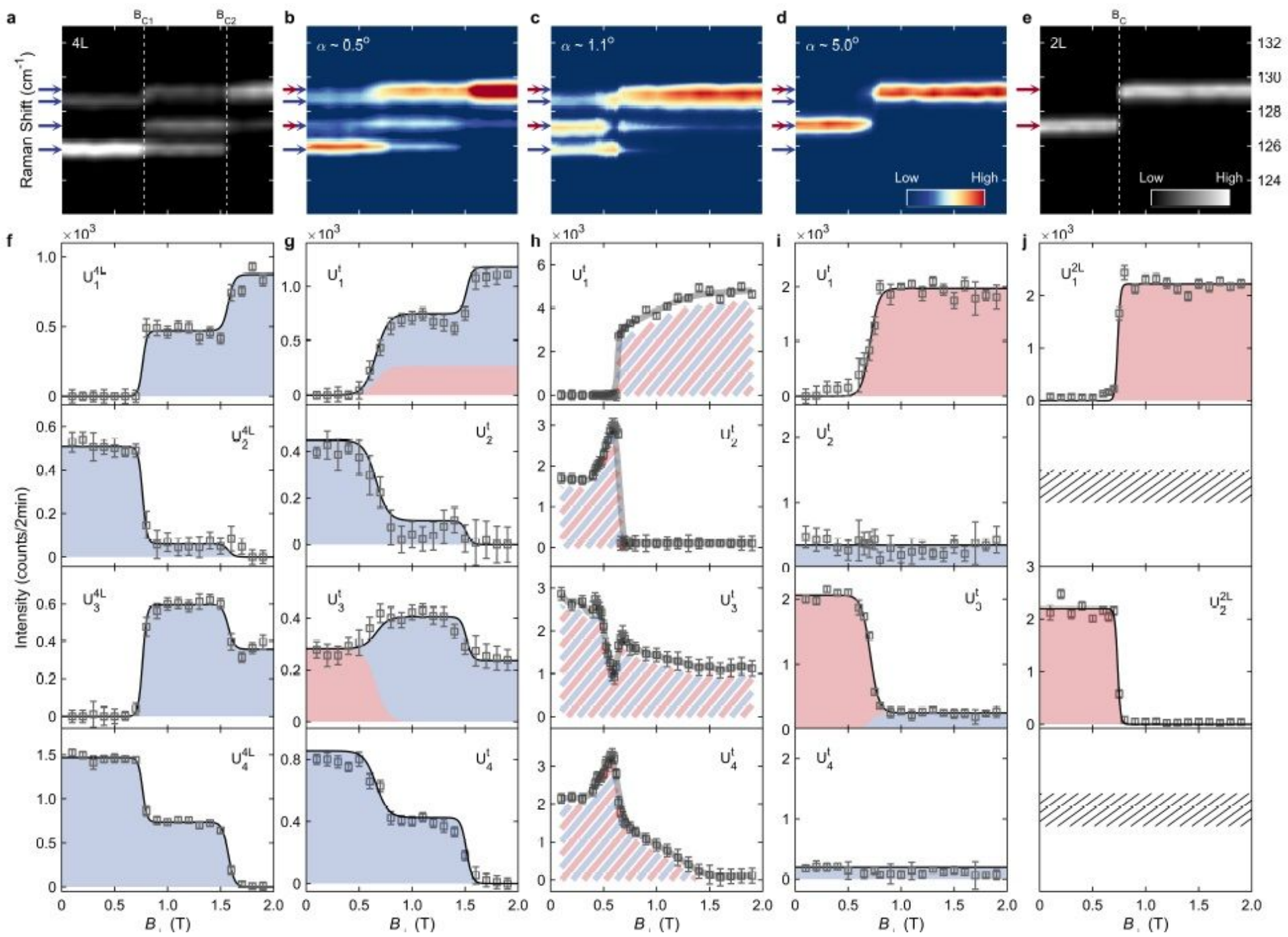

Figure 3

Magnetic field dependence of the magneto-Raman spectra of tDB Crl3 at selected twist angles. (see Manuscript file for full figure legend) 
a

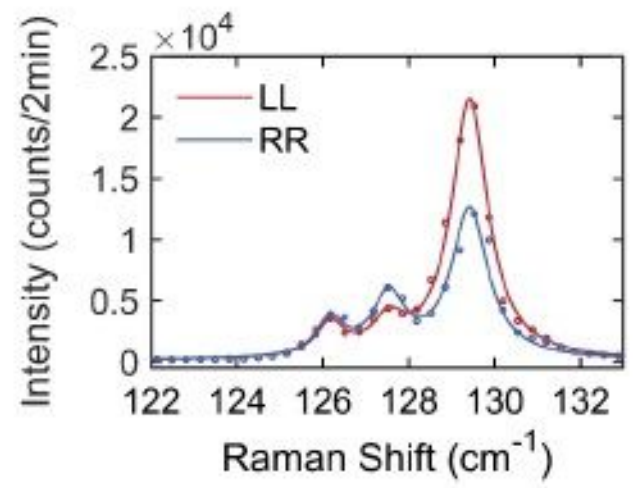

b

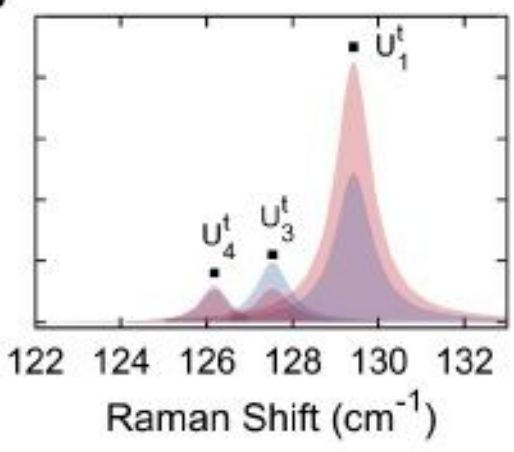

C $-\mathrm{LL}+\mathrm{B}-\mathrm{LL}-\mathrm{B}-\mathrm{RR}+\mathrm{B}-\mathrm{R}-\mathrm{R}-\mathrm{B}$
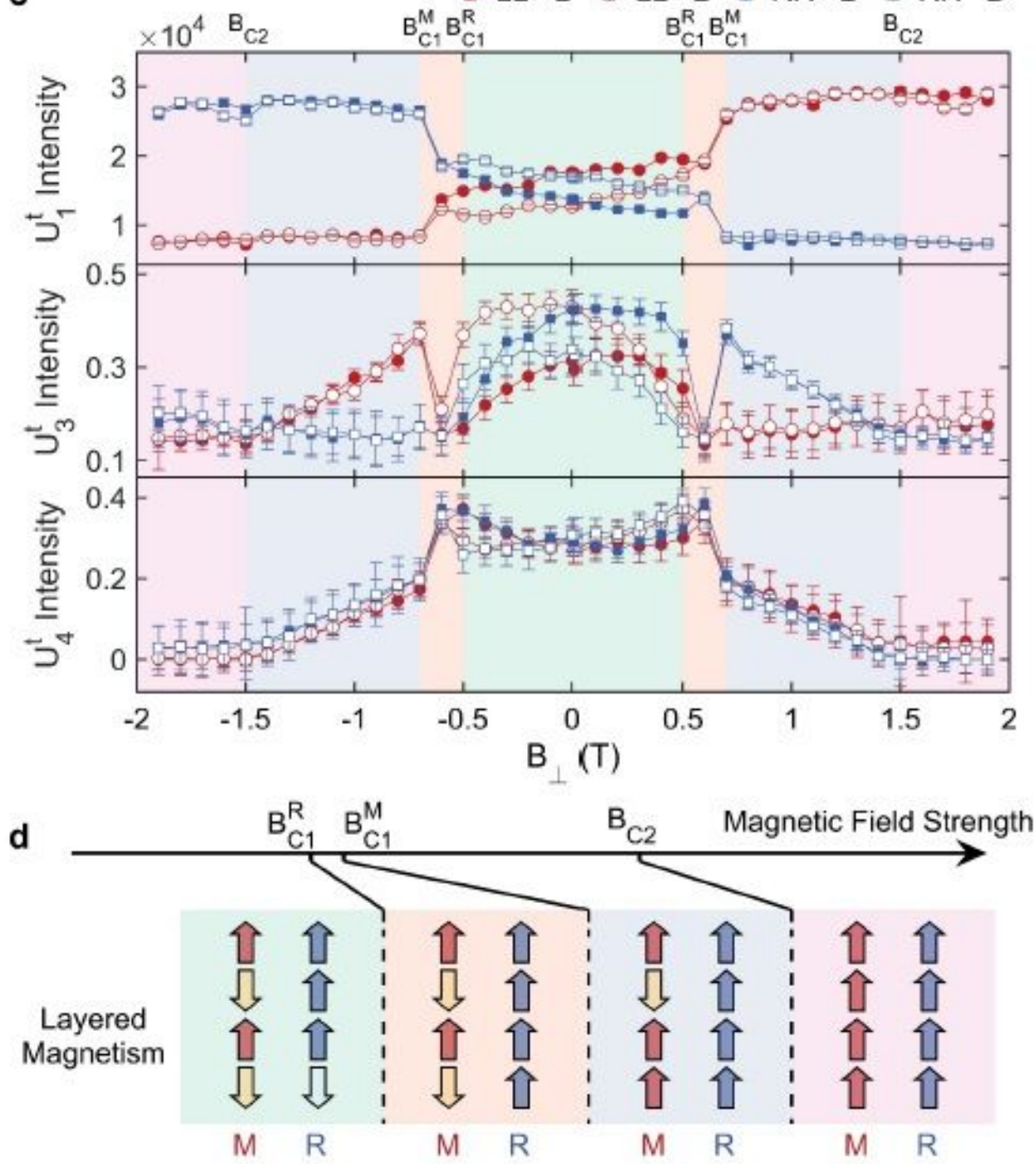

Figure 4

Magnetic circular dichroism for the 1.10 twist angle tDB Crl3. (see Manuscript file for full figure legend)

\section{Supplementary Files}

This is a list of supplementary files associated with this preprint. Click to download. 
- Supplementallnformation.pdf 